\title{
Highly efficient lipid production in the green alga Parachlorella kessleri: draft genome and transcriptome endorsed by whole-cell 3D ultrastructure
}

\author{
Shuhei Ota ${ }^{1,2+}$, Kenshiro Oshima ${ }^{3 \dagger}$, Tomokazu Yamazaki ${ }^{1,2}$, Sangwan Kim ${ }^{3,6}$, Zhe Yu $^{1}$, Mai Yoshihara', \\ Kohei Takeda ${ }^{1}$, Tsuyoshi Takeshita ${ }^{1}$, Aiko Hirata ${ }^{4}$, Kateřina Bišová ${ }^{5}$, Vilém Zachleder ${ }^{5}$, Masahira Hattori ${ }^{3}$ \\ and Shigeyuki Kawano ${ }^{1,2^{*}}$
}

\begin{abstract}
Background: Algae have attracted attention as sustainable producers of lipid-containing biomass for food, animal feed, and for biofuels. Parachlorella kessleri, a unicellular green alga belonging to the class Trebouxiophyceae, achieves very high biomass, lipid, and starch productivity levels. However, further biotechnological exploitation has been hampered by a lack of genomic information.

Results: Here, we sequenced the whole genome and transcriptome, and analyzed the behavior of P. kessleri NIES2152 under lipid production-inducing conditions. The assembly includes 13,057 protein-coding genes in a 62.5-Mbp nuclear genome. Under conditions of sulfur deprivation, lipid accumulation was correlated with the transcriptomic induction of enzymes involved in sulfur metabolism, triacylglycerol (TAG) synthesis, autophagy, and remodeling of light-harvesting complexes.
\end{abstract}

Conclusions: Three-dimensional transmission electron microscopy (3D-TEM) revealed extensive alterations in cellular anatomy accompanying lipid hyperaccumulation. The present 3D-TEM results, together with transcriptomic data support the finding that upregulation of TAG synthesis and autophagy are potential key mediators of the hyperaccumulation of lipids under conditions of nutrient stress.

Keywords: Genome, Green alga, Lipid body, Parachlorella kessleri, RNA-seq, Whole-genome sequence, Transcriptome, 3D-TEM

\section{Background}

Microalgae accumulate oil as storage lipids (TAGs), and are ideal species for developing the highly productive strains that are essential for biofuel production [1-3]. Initial efforts to exploit microalgae for biotechnology date back to approximately 140 years ago [4]. Large-scale algal cultivation has been optimized since then, but this

\footnotetext{
${ }^{*}$ Correspondence: kawano@k.u-tokyo.ac.jp

tShuhei Ota and Kenshiro Oshima contributed equally to this work

${ }^{1}$ Department of Integrated Biosciences, Graduate School of Frontier

Sciences, University of Tokyo, 5-1-5 Kashiwanoha, Kashiwa, Chiba

277-8562, Japan

Full list of author information is available at the end of the article
}

process was, in many cases, driven by trial and error, thus hindering the most effective utilization of algal potential [4]. Clearly, rapid development in this field can be achieved only through optimization of growth conditions guided by the results of basic research on algal physiology, morphology, and genomics.

The class Trebouxiophyceae is a major group in the green algal phylum Chlorophyta $[5,6]$. One of the trebouxiophycean genera, Chlorella, has been well-studied with regard to its physiology and has been exploited industrially due to its high photosynthetic growth rate and excellent biomass productivity [7-9]. Recently, Chlorella and the closely related genus, Parachlorella [10], 
have attracted attention as potential producers of triacylglycerols (TAGs) and high value-added, long-chain fatty acid feedstocks $[1,11,12]$. Recently, a new algal species closely related to Parachlorella kessleri, which is also a high lipid producer, was reported from the Indian Ocean [13]. Parachlorella kessleri, one of three commonly described Parachlorella species [10, 14], is an organism that achieves very high biomass, lipid, and starch productivities $[15,16]$. Importantly, P. kessleri is one of only a few species in which lipid productivity has been assessed not only under laboratory conditions [15-17] but also on a semi-industrial scale in outdoor photobioreactors [18], and is considered an ideal microalgal species for biofuel production.

Given their industrially verified superior growth properties, combined with high levels of lipid productivity, these species may be sustainable sources of TAG and an alternative to petroleum-based diesel fuels in the biofuel industry $[1,11]$. Of the potential TAG producers, only the genome of Nannochloropsis gaditana [19], a member of the stramenopiles [20], has been sequenced. Moreover, a species from the same genus was shown to be capable of homologous recombination, which would thus allow for efficient gene targeting [21]. Although some green algae, particularly those belonging to the genera Chlorella and Parachlorella, are more productive lipid producers than Nannochloropsis [22], only few genomic sequence have been reported (Chlorella variabilis, an endosymbiont of ciliates [23], and Chlorella protothecoides [24]), and a genome sequence of autotrophic Chlorella is currently lacking, thus limiting potential improvements in growth and productivity guided by omics techniques.

Some studies have shown that lipid content in algae can be increased by nutrient depletion [1, 4, 25-28]. Among the macronutrients (nitrogen, phosphate, and sulfur), nitrogen deprivation is widely used for stress experiments [26, 27, 29-34]. However, such stresses lower the growth rate and productivity of the system [1,35], which is a major bottleneck for producing biofuels and byproducts on commercial scales, and therefore some studies have addressed solutions using genetic engineering [36, 37]. Sulfur deprivation is an alternative stressor for the induction of starch or TAG biosynthesis $[4,16,38]$. Notably, a high starch content was maintained for a long period under conditions of sulfur starvation, suggesting that sulfur depletion is an effective method of enhancing starch productivity [4]. In contrast to Chlamydomonas [39], the cellular behavior and metabolic and transcriptomic responses under sulfur-depleted culture conditions are less well-characterized in Chlorella species. Here, we first report the reference genome of $P$. kessleri NIES-2152. Second, we also examined the transcript patterns and cellular anatomy to evaluate responses to sulfur deprivation in the induction of TAG and carbon hydrate production [4] using phenotypic assays, RNA-seq, and three-dimensional transmission electron microscope.

\section{Results and discussion}

The Parachlorella genome was sequenced and 27.8-fold pyrosequencing reads were obtained $(4,365,609$ reads in total). Reads were assembled into 5168 contigs and a total of 400 scaffolds were acquired. The present scaffolds and contigs are from nuclear, plastid, and mitochondria genomes. The total nuclear genome size was estimated to be 62.5 megabase pairs (Mbp) and 13,057 genes were identified (Table 1, Additional file 1: Figures S1 and S2). Thirty major scaffolds cover $48.8 \%$ of the genome. Of the annotated genes (Additional file 1: Table S1), $49.7 \%$ of their proteins were associated with Kyoto encyclopedia of genes and genomes (KEGG) orthology numbers.

To characterize the phenotype and transcriptome under sulfur deprivation, P. kessleri was batch-cultivated under continuous light (LL) and sulfur replete and deplete $( \pm S)$ conditions. Growth was determined as biomass dry weight as well as cell density. As expected, growth was restricted under sulfur-depleted conditions, in contrast to its growth in tris-acetate-phosphate (TAP) medium, where a logarithmic growth phase was clearly observed (Fig. 1a). Higher starch contents per cell were observed after 2 days in culture under conditions of sulfur deprivation (6.19- and 6.62-fold changes on days 2

Table 1 The Parachlorella genome assembly statistics

\begin{tabular}{ll}
\hline Characteristic & \\
\hline Genome size & $62.5 \mathrm{Mbp}$ \\
GC (\%) & $58.30 \%$ \\
Number of scaffolds & $400 \mathrm{scaffolds}$ \\
Average of scaffold size & $156,382 \mathrm{bp}$ \\
N50 scaffold size (>2k bases: 400 scaffolds) & $543,086 \mathrm{bp}$ \\
N50 scaffold size (>5k bases: 213 scaffolds) & $595,262 \mathrm{bp}$ \\
N50 scaffold size (>10k bases: 193 scaffolds) & $595,262 \mathrm{bp}$ \\
Longest scaffold size & $2,165,932 \mathrm{bp}$ \\
Number of contigs & $5168 \mathrm{contigs}$ \\
Average of contig size & $11,748 \mathrm{bp}$ \\
N50 contig size (>500 bases: 5168 contigs) & $32,688 \mathrm{bp}$ \\
N50 contig size (>5k bases: 2326 contigs) & $36,671 \mathrm{bp}$ \\
N50 contig size (>10k bases: 1643 contigs) & $40,504 \mathrm{bp}$ \\
Longest contig size & $198,966 \mathrm{bp}$ \\
Numbers of genes & $13,057 \mathrm{genes}$ \\
Average of protein length & $467.0 \mathrm{aa}$ \\
Average gene density & $4.8 \mathrm{~kb} / \mathrm{gene}$ \\
Average number of exons per gene & $7.9 \mathrm{exons}$ \\
Average exon length & $176.3 \mathrm{bp}$ \\
Average coding sequence & $29.30 \%$ \\
\hline
\end{tabular}


and 3, respectively) (Fig. 1b), consistent with results from the previous sulfur-depletion study [38].

There was a remarkable difference in total lipid content between $\pm S$ cultures. After 5 days of cultivation, P. kessleri growing in dSTAP (sulfur-deprived TAP) reached a yield of $0.25 \mathrm{mg} \mathrm{mL}^{-1}$ total lipids, whereas $P$. kessleri in TAP gradually accumulated total lipids over a 10-day period of batch cultivation, but achieved a yield of only $0.14 \mathrm{mg} \mathrm{mL}^{-1}$ total lipid (Fig. 1c). This finding indicates that total lipid yield was accelerated under sulfur deprivation, in contrast to cultivation in TAP medium, so that in the 5-day-old, sulfur-deprived culture, the lipid content represented up to $50.7 \%$ of the dry weight (Fig. 1d).

A previous report showed that lipid accumulation under nitrogen-limited conditions was tightly connected with cellular processes that are related to lipogenesis, macromolecule metabolism, and autophagy in a lipidproducing yeast [40]. Here, we focus on the autophagyrelated genes to show the transcriptome dynamics. During sulfur deprivation, some autophagy-related genes were upregulated in the early and late logarithmic-phase cultures (Fig. 1e). However, no genes were downregulated in the stationary-phase culture, except for ATG3, ATG5, and beclin 1, suggesting that autophagy and recycling of subcellular components are enhanced under sulfur-depleted conditions in the early and late logarithmic phases.

Transmission electron microscopy allows high-resolution imaging to study cellular anatomy and ultrastructure, which is inaccessible by other techniques. This is essential when studying cells in which one of the organelles is enlarged, covering other compartments and thus more or less blocking their observation by other means. Examples of such situations are cells over-producing astaxanthin [41] or lipids [42]. The main limitation of TEM is that it traditionally facilitates only two-dimensional imaging of approximately $80 \mathrm{~nm}$ ultrathin sections. This can be overcome by analyzing sequential sections of a single cell stacked together using three-dimensional transmission electron microscopy (3D-TEM) technology, leading to a three-dimensional image of cell ultrastructure with very high resolution. The technique was used here to study lipid hyperaccumulation within P. kessleri cells in the stress experiment.

Representative cells from three phases-logarithmic growth (control), starch-rich, and lipid-rich, were analyzed using 3D-TEM (Fig. 2, Additional files 2, 3, 4 and 5). In the logarithmic growth phase, little starch was accumulated and no lipid bodies were found (Fig. 2a-c, Additional file 2). The chloroplast and mitochondria accounted for 38.5 and $5.9 \%$ of the relative volume, respectively (Fig. 2j). In the starch-rich phase, many starch grains were observed in the chloroplasts (Fig. $2 \mathrm{~d}-\mathrm{f}$,
Additional file 3), accounting for $9.7 \%$ of the relative volume (Fig. 2k), whereas lipid bodies showed less accumulation ( $0.4 \%$ of the relative volume). In this phase, the relative volume of the chloroplasts was $34.3 \%$ (Fig. $2 \mathrm{k}$ ).

In the lipid-rich phase, the ultrastructure and volume of the subcellular components were dramatically changed (Fig. 2g-i, Additional files 4 and 5). The most remarkable change from the starch-rich stage to the lipid-rich stage was the relative volume of chloroplasts and lipid bodies (Fig. 2i). The chloroplast was highly degenerate and its relative volume was reduced almost tenfold to $4.4 \%$ (Fig. 2l); it was also re-located to one side of the cell periphery (Fig. 2g, Additional file 4). Concurrently, lipid bodies over-accumulated and accounted for $51.7 \%$ of the relative volume (Fig. 2l).

Compared to the morphological changes in Chlorella sorokiniana under nutrient stress [42], P. kessleri possessed larger lipid bodies and these accounted for a considerable portion of the cell. This was accompanied by degradation of the chloroplast in the lipid-rich phase of P. kessleri growth (Fig. 2g, Additional files 4 and 5), but not in C. sorokiniana. This reflects the main differences between P. kessleri and other Chlorella species with respect to lipid production. The subcellular degradation process probably, and at least in part, involves autophagy through upregulation of ATG-related transcripts, thus enabling recycling of subcellular compartments and increased lipid accumulation.

Levels of individual transcripts were expressed in a heat map and subsequently applied to a metabolic pathway map (Fig. 3, Additional file 6) and KEGG category analysis (Additional file 1: Figures S3-S5). Overall, the expression of genes involved in the Calvin-Benson, tricarboxylic acid (TCA), glyoxylate, and C4 dicarboxylic acid cycles showed decreased expression under sulfurdepleted conditions. Other metabolic pathways, such as fatty acid metabolism, autophagy, TAG, cysteine, and methanethiol synthesis were transcriptionally upregulated (Fig. 3). KEGG category analysis also indicated that cysteine and methionine metabolism (category 3: minor function category) were upregulated during the entire cultivation period under sulfur deprivation (Additional file 1: Figure S4).

Under sulfur deprivation, the sulfate transporter gene $\left(10836 \_t\right)$ was highly upregulated $\left(\log _{2}\right.$ value $=6.6$ in the late logarithmic phase, $P<0.0005)$, together with cysteine dioxygenase (6136_t) $\left(\log _{2}\right.$ value $=2.5$ and 5.6 in the late logarithmic and stationary phases, respectively; $P<0.05$ at stationary phase) (Additional file 7). This is in agreement with the induction of transcripts associated with sulfur acquisition and assimilation, synthesis of sulfurcontaining amino acids, cysteine degradation, and sulfur recycling, as shown in Chlamydomonas [39]. In addition, 

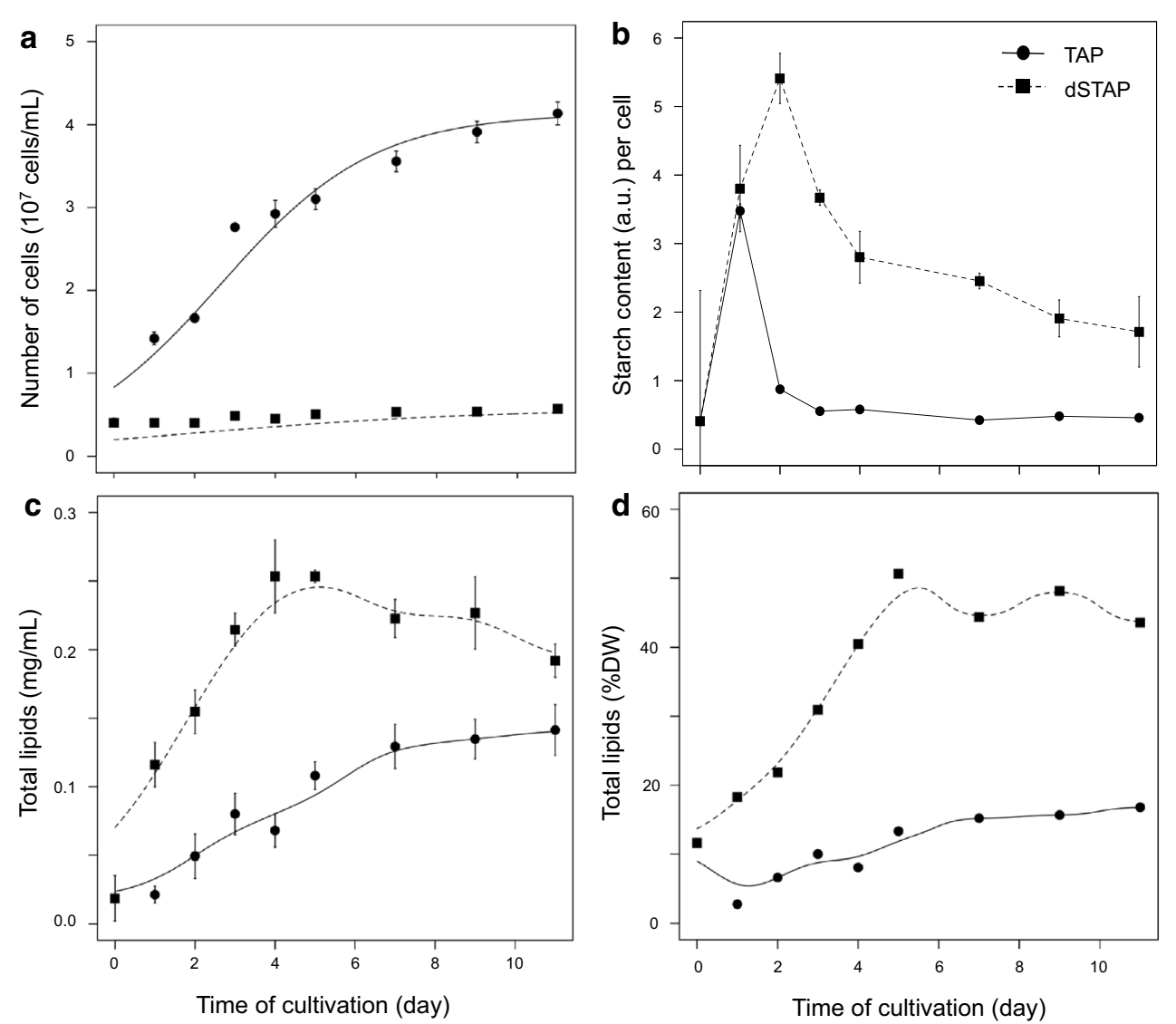

e

$\log _{2}(\mathrm{dSTAP} / \mathrm{TAP})$

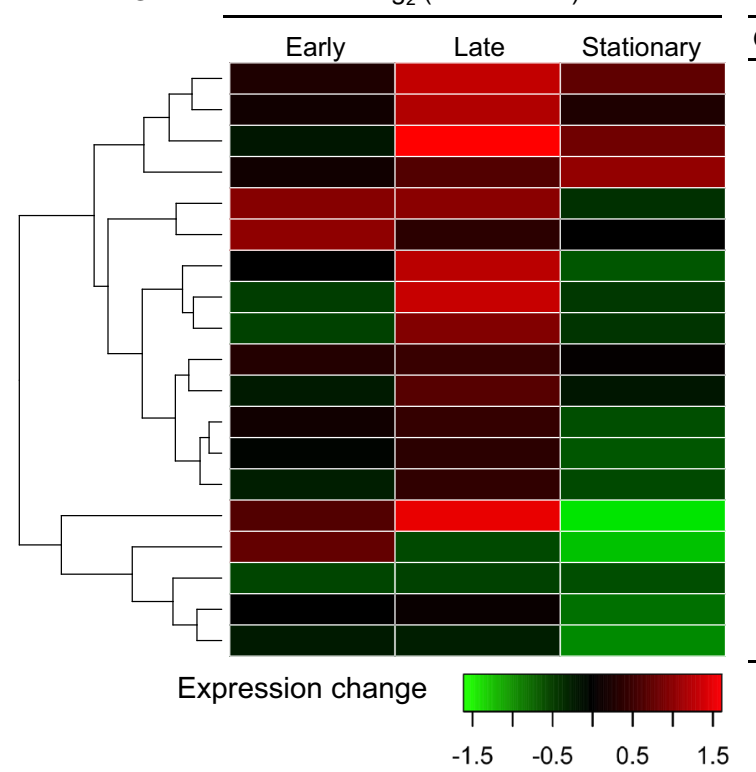

\begin{tabular}{|c|c|}
\hline Genome ID & Description \\
\hline$\overline{155 \_t}$ & Beclin 1 \\
\hline $7935 \mathrm{t} t$ & Autophagy-related protein 4 \\
\hline $4380-\mathrm{t}$ & Autophagy-related protein 5 \\
\hline $9402 \mathrm{t}$ & Autophagy-related protein 3 \\
\hline $7174 \_\mathrm{t}$ & V-type proton ATPase catalytic subunit A-like \\
\hline $8008+\mathrm{t}$ & Autophagy-related protein 12 \\
\hline $2754 \_\mathrm{t}$ & Apg1/Unc-51-like serine-threonine kinase \\
\hline $6863 \_t$ & Autophagy-related protein 7 \\
\hline $892 \_t$ & Phosphatidylinositol 3-kinase \\
\hline 7007_t & Phosphoinositide-3-kinase \\
\hline 12392 t & V-type $\mathrm{H}+$-transporting ATPase subunit I \\
\hline $7173 \_t$ & V-type $\mathrm{H}$-transporting ATPase subunit A \\
\hline $4345+\mathrm{t}$ & 5'-AMP-activated protein kinase \\
\hline $7175 \_\mathrm{t}$ & V-type $\mathrm{H}+$-transporting ATPase subunit A \\
\hline $7893 \_\mathrm{t}$ & Autophagy-related protein 8 \\
\hline $7418 \_t$ & 5'-AMP-activated protein kinase \\
\hline 10798 _t & 5'-AMP-activated protein kinase \\
\hline $11799+\mathrm{t}$ & Autophagy-related protein 101-like \\
\hline $12432 \mathrm{t}$ & Os06g0267600; Autophagy-related \\
\hline
\end{tabular}

$$
\begin{array}{llll}
-1.5 & -0.5 & 0.5 & 1.5
\end{array}
$$

Fig. 1 Phenotypic and transcriptomic response to sulfur deprivation. a Average \pm SD $(n=3)$ growth in TAP (circles) and dSTAP (squares). b Time course of average $\pm \mathrm{SD}(n=3)$ starch accumulation. c Time course of average \pm SD total lipid accumulation. $\mathbf{d}$ Time course of average $(n=3)$ total lipid yield (\% DW). e Heat map of relative expression levels of autophagy-related genes. Values are indicated as the fold change (log ${ }_{2}$-ratio) of transcript levels under sulfur-deprived conditions relative to sulfur-replete conditions for the three culture phases (early and late log phases and stationary phase). The dendrogram represents hierarchical clustering based on expression values. TAP (solid line) and dSTAP (dashed line) lines are fitted curves as described in "Methods". Real values and descriptions of autophagy-related genes are as in Additional file 7 


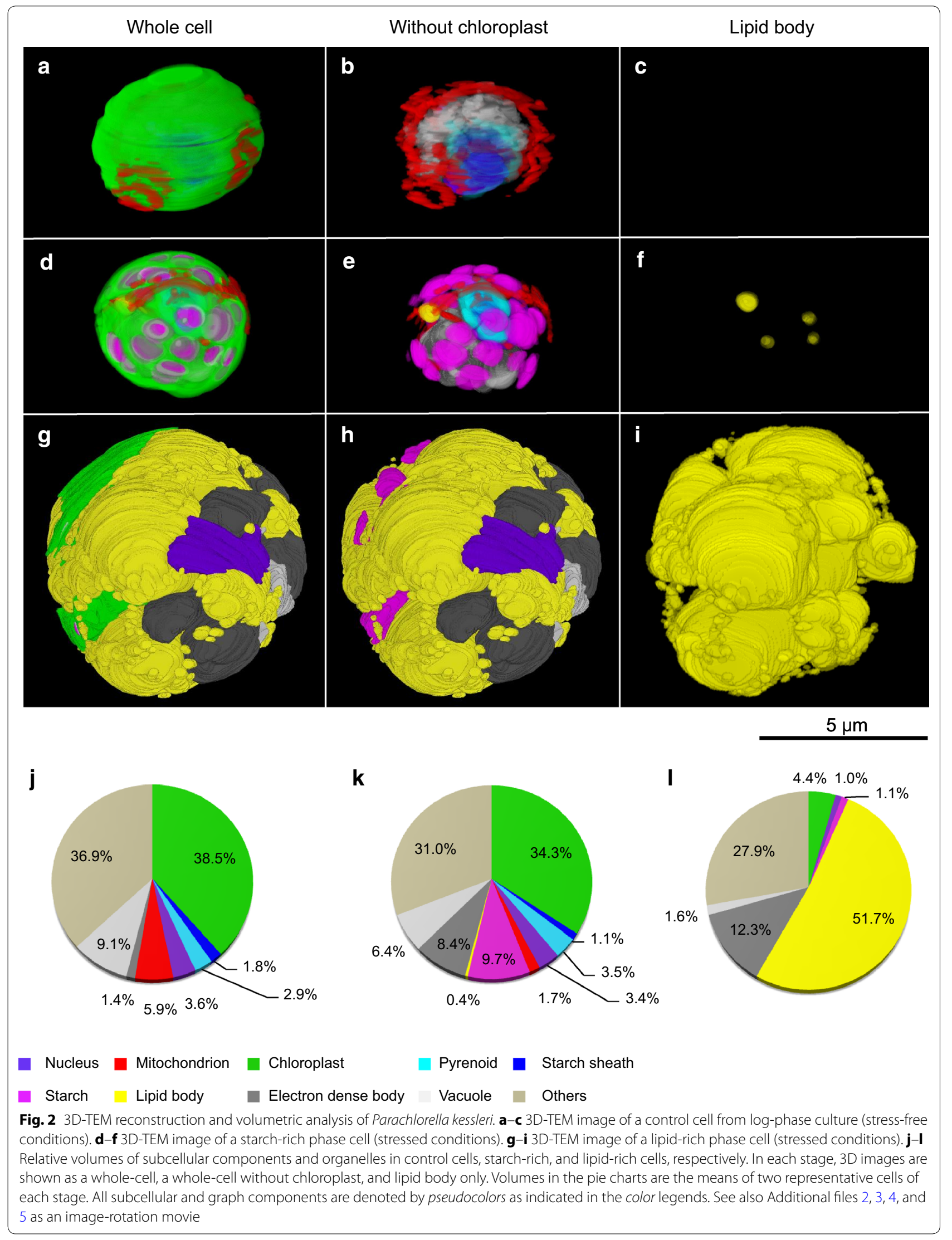




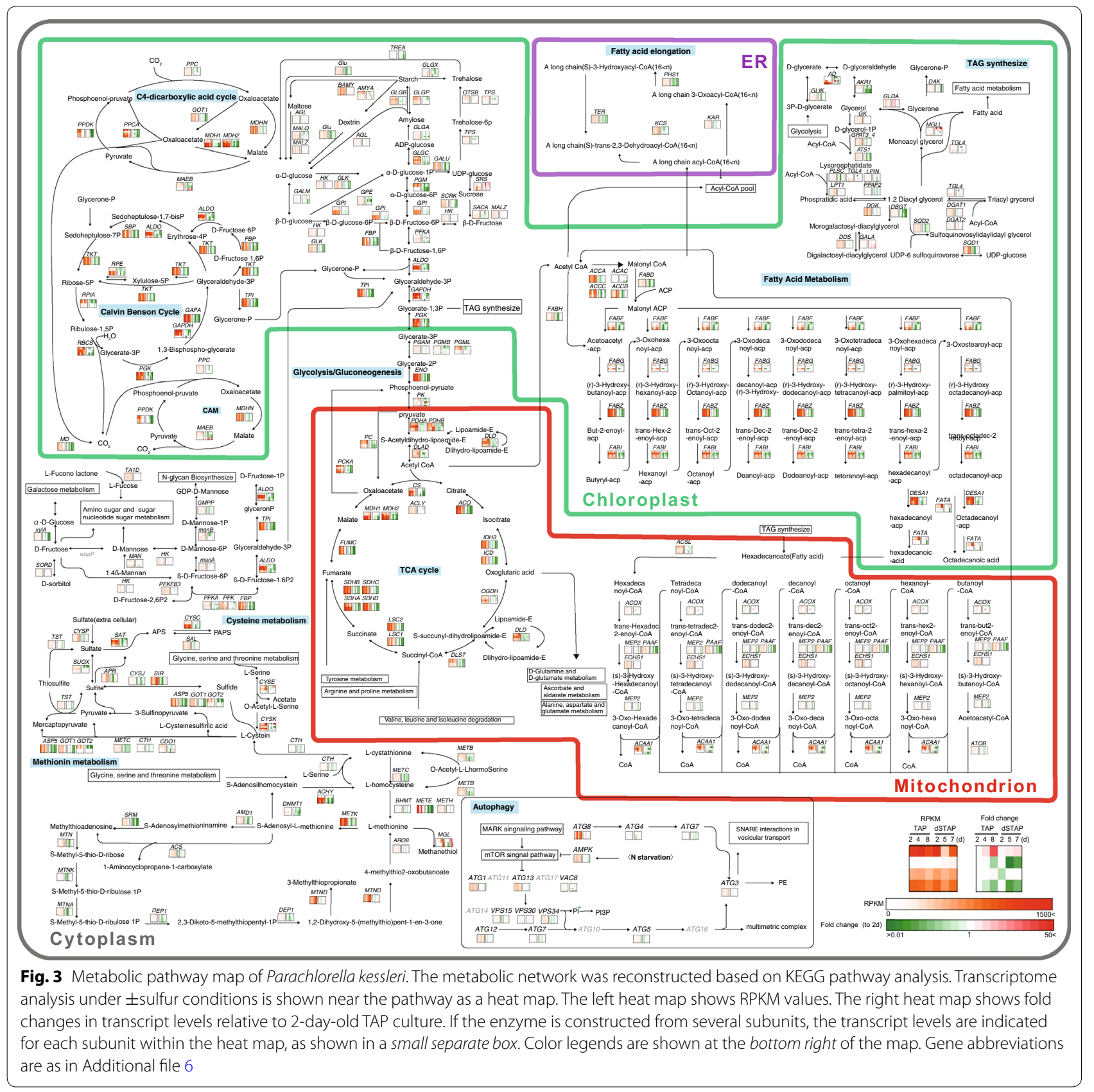

the gene for methionine-gamma-lyase (MGL) was highly upregulated (Fig. 3). Degradation of L-methionine is catalyzed by MGL, resulting in methanethiol production. Because methanethiol is a sulfur-containing compound, we suspect that this metabolic pathway may be used for recycling of sulfur under sulfur-limited conditions.

It should be emphasized that transcripts responsive to sulfur deprivation also included genes for the lightharvesting complex of PSII (LHCB) (Additional file 7). Almost all genes encoding LHCBs were downregulated or did not show varying levels of expression between the $\pm \mathrm{S}$ conditions; only one gene, LHCB1 (1779_t), was strongly upregulated $\left(P<3 \times 10^{-7}\right)$. Compared to the no-stress condition, transcript levels of $L H C B 1$ under sulfur-depleted conditions increased by 2050- and 5106-fold in the late logarithmic and stationary phases, respectively. LHCB1 (1779_t) is a homolog of LHCBM9 in Chlamydomonas (JGI accession no: 184479). In Chlamydomonas [39, 43], LHCBM9 was upregulated during sulfur deprivation. In the RNA-seq analysis [39], 
LHCBM9 transcripts, which were barely detectable when cells were grown in $+\mathrm{S}$ medium, were upregulated by $>1000$-fold during sulfur deprivation, whereas transcript levels of the other $L H C B M s$ declined, similarly to P. kessleri (Additional file 7). Because LHCBM9 contains at least three fewer sulfur-containing amino acids than other LHCBMs, it was suggested that exchange for LHCBM9 might enable recycling of sulfur-containing amino acids and their reallocation among other proteins with low sulfur amino acid contents. This process may represent an extreme measure for sulfur use that could extend cell viability under sulfur-depleted conditions. This notion was further supported by upregulation of some autophagy-related genes in the early and late logarithmic phases of sulfur-depleted cultures (Fig. 1e).

Considering transcriptionally upregulated metabolisms that involve hyperaccumulation of lipids, TAG synthesis (e.g., DGAT1) and autophagy emerge as candidates for the key mediator of lipid hyperaccumulation under stress conditions. The present observation based on the 3D-TEM data demonstrates that hyperaccumulation of lipids and high degradation of the chloroplast occur concurrently under stress conditions. These results illustrate that analysis based on 3D-TEM and phenotypic assays confirm the finding that the upregulation of a key mediator is involved in hyperaccumulation of lipids under nutrient stress conditions in P. kessleri.

Overall, this study supports the notion that basic research is required to accelerate development in the field of algal biotechnology. The genomic information on the industrially verified, biomass-producing algal species $P$. kessleri provided here will facilitate both basic and applied research not only in the field of algal-derived biofuels but will also serve as a foundation for future genetic manipulation of the TAG biosynthetic pathway (and others) in this species. Similarly, the 3D-imaging approach is a promising tool for analysis of global ultrastructural changes in whole-cells.

\section{Conclusions}

We sequenced and analyzed the whole genome of $P$. kessleri, a high-biomass lipid-rich green alga related to Chlorella. Transcriptomic analysis suggested that lipid accumulation under conditions of sulfur depletion is associated not only with the induction of sulfur metabolism but also TAG synthesis, light-harvesting complexes, and autophagy. Moreover, the metabolic changes under \pm sulfur conditions, autophagy-like changes in cell anatomy following lipid accumulation, were visualized by 3D-TEM ultrastructural analysis. The Parachlorella genome information provided by this study will facilitate basic research and further analysis of applied phycology, in addition to potential genetic manipulation, of the TAG biosynthesis pathway in this industrially verified biomass-producing species.

\section{Methods}

\section{Growth conditions and DNA and RNA extraction}

Parachlorella kessleri NIES-2152 was obtained from the National Institute for Environmental Studies in Tsukuba, Japan. For genomic DNA extraction, P. kessleri cells were grown at $20{ }^{\circ} \mathrm{C}$ under $70 \mu \mathrm{mol}$ photons $\mathrm{m}^{-2} \mathrm{~s}^{-1}$ for $12 \mathrm{~h}: 12 \mathrm{~h}=$ light (L):dark (D) cycle at $20{ }^{\circ} \mathrm{C}$ in TAP medium (Additional file 8: Table S2). Cells from 2-week-old cultures were harvested by centrifugation at $2500 \mathrm{~g}$ for $10 \mathrm{~min}$ at room temperature (r.t.), and the resulting pellet (10 g of wet weight) was immediately frozen with liquid nitrogen. DNA extraction was performed using the DNeasy Plant Maxi Kit (Qiagen, Hilden, Germany) according to the manufacturer's protocol. For RNA extraction, cells were grown in TAP or dSTAP (Additional file 8: Table S3) medium in a $500 \mathrm{ml}$ flask (Iwaki, Tokyo, Japan) under $100 \mu \mathrm{mol}$ photons $\mathrm{m}^{-2} \mathrm{~s}^{-1}$ for $12 \mathrm{~h}: 12 \mathrm{~h}=$ light (L):dark (D) cycle at $20{ }^{\circ} \mathrm{C}$. Cells from 2 -week-old cultures were harvested by centrifugation at $2500 \mathrm{~g}$ for $10 \mathrm{~min}$ at r.t., and the resulting pellet was immediately frozen with liquid nitrogen. Total RNA extraction was performed using the Sepasol ${ }^{\circledR}$-RNA I Super G kit (Nacalai Tesque, Kyoto, Japan) followed by poly (A) mRNA purification with Dynabeads Oligo (dT) (Life Technologies, Carlsbad, USA) in cultures grown under conditions of \pm sulfur in the early (2 days old) and late (4-5 days old) logarithmic phases and stationary phase (7-8 days old). In this experiment, $12.5 \mathrm{~mL}$ of the Sepasol ${ }^{\circledR}$-RNA I Super G for every $0.5 \mathrm{~g}$ of cells were used in each experimental group. The purification was performed in three rounds. In the first round, $150 \mu \mathrm{g}$ of total RNA were used for mRNA purification using $1 \mathrm{mg}$ of Dynabeads Olig (dT) according to the manufacturer's protocol with modifications. Finally, $500 \mathrm{ng}$ of mRNA were obtained.

\section{Genomic and mRNA sequences and annotation}

The genomic sequence of $P$. kessleri NIES-2152 was determined using 454 pyrosequencing for single-end (SE) and paired-end (PE, 8 kb-span library) data. We generated $3,561,169$ reads by $\mathrm{SE}$ and 804,440 reads by $\mathrm{PE}$, which provided 27.8-fold coverage of the genome. The assembly of the obtained sequence data resulted in the generation of 400 scaffolds using Newbler version 2.8 (Roche, Branford, CT, USA). The total scaffold length was $2,653,566$ bp with a $\mathrm{G}+\mathrm{C}$ content of $58.4 \%$. Proteinencoding regions were predicted using the GeneMarkES [44]. We annotated 13,057 predicted genes using the BLASTP program [45] (e-value cut-off of $1 \mathrm{E}-5$ ) with the NCBI-nr and KEGG databases. 
The mRNA sequencing libraries of $P$. kessleri NIES2152 were constructed using the ion total RNA-Seq Kit v2 (Life Technologies, Carlsbad, USA) and the libraries were sequenced using an Ion PGM sequencer (Life Technologies, Carlsbad, USA). We generated 4,220,822 reads (2d: 2-day-old culture in TAP), 5,681,304 reads (4d: 4-day-old culture in TAP), 4,742,155 reads (8d: 8 -day-old culture in TAP), 4,144,745 reads (ds_2d: 2-day-old culture in dSTAP), 4,220,451 reads (ds_5d: 5-day-old culture in dSTAP), and 3,694,851 reads (ds_7d: 7-day-old culture in dSTAP) from six mRNA samples. The Parachlorella gene IDs and full descriptions of protein names are given in Additional files 6 and 7.

\section{Reconstruction of KEGG pathway map and mRNA expression analysis}

The RNA-seq reads were mapped to the predicted genes using Newbler v.2.8. Comparative expression analyses were performed using standardized reads per kilobase of exon per million mapped sequence reads (RPKM) values. Functional annotation descriptions were assigned by BLASTP [45] with the KEGG database (e-value cutoff of $1 E-10)$. The metabolic network was reconstructed using KEGG mapper (http://www.genome.jp/kegg/) with the $\mathrm{KO}$ numbers as objects shown in Additional file 6 . The resulting KEGG map was redrawn manually using Adobe Illustrator v. 16.0.4 (Adobe Systems). An abbreviated gene names are given in Additional file 6. A heat map of the autophagy-related transcriptomes was generated using the heatmap 2 function from the Gplots package v. 2.16.0 in the $\mathrm{R}$ statistical software v. 3.1.0 (http://www.R-project.org/). Statistical testing for gene expression was performed in $\mathrm{R}$ with DESeq [46] using the no replicate method. The $P$ values are provided in Additional file 9. We performed KEGG category analysis in which different sequences were treated as different genes.

\section{Phenotypic assays for biomass and lipid production}

Pre-cultures were grown under $100 \mu \mathrm{mol}$ photons $\mathrm{m}^{-2} \mathrm{~s}^{-1}$ for $12 \mathrm{~h}: 12 \mathrm{~h}=\mathrm{L}: \mathrm{D}$ cycle at $23{ }^{\circ} \mathrm{C}$. Cells of 4 -dayold cultures were centrifuged at $1500 \mathrm{~g}$ for $5 \mathrm{~min}$ at r.t. and collected as a pellet. The pellet was re-suspended and inoculated into $500 \mathrm{~mL}$ of TAP or dSTAP medium (the initial concentration of the culture was $\sim 7 \times 10^{6}$ cells $\left.\mathrm{mL}^{-1}\right)$. The batch cultures were grown under continuous illumination $\left(100 \mu \mathrm{mol}\right.$ photons $\left.\mathrm{m}^{-2} \mathrm{~s}^{-1}\right)$ at $21-23^{\circ} \mathrm{C}$ and agitated with a magnetic stirrer (MGM-66, Shibata, Tokyo, Japan) at 100-150 rpm.

Cells were counted using a particle counter (CDA1000, Sysmex, Kobe, Japan). A general linear model was fit to values of the cell numbers in each condition for growth curves. For dry weight determination, an aliquot of cell culture was sampled into a pre-weighed sampling tube and centrifuged at $6000 \mathrm{~g}$ for $5 \mathrm{~min}$ at r.t. The supernatant was then removed and the cell pellet was dried for at least $3 \mathrm{~h}$ to a constant weight at $105{ }^{\circ} \mathrm{C}$. The sampling tube was weighed using a precision analytical balance (NewClassic MS, Mettler Toledo, MD). For total lipid extraction and measurements, we used a previously described method [16]. Briefly, total lipids were extracted using methyl-tertbutyl ether (MTBE) [47], and the weight of total lipids was measured gravimetrically using a precision analytical balance. Starch content was quantified using the Lugol staining method as described [48]. Growth curves were estimated using a general linear model (glm function), and other curves (except for starch assay) were fitted using the ksmooth function in R statistical software v. 3.1.0.

\section{D-TEM analysis}

We observed three representative stages: control (4 days old in TAP under LD), starch-rich [6 days old in dSTAP under light/dark cycle (LD)], and lipid-rich (6 days old in dSTAP under LL). Cells at each stage were pre-fixed for $2 \mathrm{~h}$ with $2.5 \%$ glutaraldehyde, post-fixed with $1 \%$ $\mathrm{OsO}_{4}$ for $2 \mathrm{~h}$ at r.t., and then rinsed with $0.05 \mathrm{M}$ sodium cacodylate buffer ( $\mathrm{pH}$ 7.2). The fixed cells were then dehydrated using a graded ethanol series, incubated in ethanol:acetone $=1: 1$ and finally suspended in $100 \%$ acetone at r.t. The dehydrated samples were infiltrated with increasing concentrations of Spurr's resin [49] in acetone and finally with $100 \%$ Spurr's resin. Ultrathin serial sections were cut on a Reichert Ultracut S ultra-microtome (Leica, Vienna, Austria) using a diamond knife. Serial sections were mounted on copper grids coated with polyvinyl formvar films and stained in $3 \%$ aqueous uranyl acetate and lead citrate [50]. The sections were observed at $100 \mathrm{kV}$ using an $\mathrm{H}-7650$ transmission electron microscope (Hitachi High Technologies, Tokyo, Japan).

3D-TEM imaging followed a previously described method [41]. Briefly, contours of each subcellular element (e.g., nucleus, chloroplast, lipid body) were traced manually. After binarization of the traced subcellular elements, 3D images were reconstructed using TRI/3D SRFIII software (Ratoc System Engineering, Tokyo, Japan). Voxel-based volumetric analyses were performed using the TRI/3D SRFIII system and data were presented as the means of two representative cells.

\section{Nucleotide sequence accession numbers}

The P. kessleri NIES-2152 whole genome has been deposited in DDBJ/EMBL/GenBank under the accession BBXU01000001-BBXU01003651 (BioProject number: PRJDB3487). 


\section{Additional files}

Additional file 1: Figure S1. Number of genes versus size (nt in length) of genes. Figure S2. Number of genes versus number of exons. Figure S3. KEGG category analysis in energy metabolism. Figure S4. KEGG category analysis in amino acid metabolism. Figure S5. KEGG category analysis in lipid metabolism. Table S1. Percentage of genes annotated.

Additional file 2. 3D-reconstruction movie (mov format) of a control cell without a chloroplast. The color legends used in the movie are the same as in Fig. 2

Additional file 3. 3D-reconstruction movie (mov format) of a starch-rich cell without a chloroplast. The color legends used in the movie are the same as in Fig. 2.

Additional file 4. 3D-reconstruction movie (mov format) of a lipid-rich cell. The color legends used in the movie are the same as in Fig. 2.

Additional file 5. 3D-reconstruction movie (mov format) of lipid bodies in a lipid-rich cell.

Additional file 6. Gene ID, KO numbers, and full description of gene names corresponding to the metabolic pathway map.

Additional file 7. RPKM and fold change of all Parachlorella transcripts.

Additional file 8: Table S2. Recipe of TAP medium. Table S3. Recipe of dSTAP medium.

Additional file 9. $P$ values of the present RNA-seq analysis.

\section{Abbreviations}

dSTAP: sulfur-deprived TAP; KEGG: Kyoto encyclopedia of genes and genomes; LD: light/dark cycle; LL: continuous light; NIES: National Institute for Environmental Studies, Tsukuba, Japan; RPKM: reads per kilobase of exon per million mapped sequence reads; TAG: triacylglycerol; TAP: tris-acetate-phosphate; TCA: tricarboxylic acid; 3D-TEM: three-dimensional transmission electron microscopy.

\section{Authors' contributions}

SO cultured P. kessleri and prepared samples for each experiment, and drafted the manuscript. SO and TT extracted genomic DNA, and TY extracted total RNA and purified mRNA. KO sequenced genomic DNA and mRNA, assembled the scaffolds, and implemented the automated annotation of the genome. KO, SaK, and ZY performed bioinformatic analysis. TY, KT, and ZY analyzed metabolic pathways. SO, MY, and AH performed the experiments for growth, lipid, and starch assays and for 3D-TEM imaging. ShK and MH conceived of the study, and participated in the planning and coordination of the study. KO, TY, $\mathrm{KB}, \mathrm{VZ}$ and ShK participated in the design of the study and helped to revise the manuscript. All authors read and approved the final manuscript.

\section{Author details}

1 Department of Integrated Biosciences, Graduate School of Frontier Sciences, University of Tokyo, 5-1-5 Kashiwanoha, Kashiwa, Chiba 277-8562, Japan. 2 Japan Science and Technology Agency (JST), CREST, 5-1-5 Kashiwanoha, Kashiwa, Chiba 277-8562, Japan. ${ }^{3}$ Center for Omics and Bioinformatics, Graduate School of Frontier Sciences, University of Tokyo, 5-1-5 Kashiwanoha, Kashiwa, Chiba 277-8561, Japan. ${ }^{4}$ Bioimaging Center, Graduate School of Frontier Sciences, University of Tokyo, 5-1-5 Kashiwanoha, Kashiwa, Chiba 277-8562, Japan. ${ }^{5}$ Institute of Microbiology, CAS, Centre Algatech, Laboratory of Cell Cycles of Algae, Opatovický mlýn, 37981 Třeboň, Czech Republic. ${ }^{6}$ Present Address: Department of Genetic Resources Technology, Faculty of Agriculture, Kyushu University, Fukuoka, Japan.

\section{Acknowledgements}

We thank Yuko Yoshizawa, Rika Shinohara, Yukiko Kudo, and Minako Miki (University of Tokyo) for their technical assistance with this research and 3D-TEM analysis. We also thank Erica lioka, Misa Kiuchi, Hiromi Kuroyanagi, and Emi Omori (University of Tokyo) for their technical help with sequencing. This research was supported with funding provided by the Japan Science and
Technology Agency (JST) in the CREST program (to ShK). We also acknowledge the Cross-ministerial Strategic Innovation Promotion Program (SIP), Japan.

\section{Competing interests}

The authors declare that they have no competing interests.

Received: 2 July 2015 Accepted: 5 January 2016

Published online: 25 January 2016

\section{References}

1. Hu Q, Sommerfeld M, Jarvis E, Ghirardi M, Posewitz M, Seibert M, Darzins A. Microalgal triacylglycerols as feedstocks for biofuel production: perspectives and advances. Plant J. 2008;54:621-39.

2. Georgianna DR, Mayfield SP. Exploiting diversity and synthetic biology for the production of algal biofuels. Nature. 2012;488:329-35.

3. Markou G, Nerantzis E. Microalgae for high-value compounds and biofuels production: a review with focus on cultivation under stress conditions. Biotechnol Adv. 2013:31:1532-42.

4. Vítová M, Bišová K, Kawano S, Zachleder V. Accumulation of energy reserves in algae: from cell cycles to biotechnological applications. Biotechnol Adv. 2015;33:1204-18.

5. Friedl T. Inferring taxonomic positions and testing genus level assignments in coccoid green lichen algae: a phylogenetic analysis of $18 \mathrm{~S}$ ribosomal RNA sequences from Dictyochloropsis reticulata and from members of the genus Myrmecia (Chlorophyta, Trebouxiophyceae cl. nov). J Phycol. 1995;31:632-9.

6. Lewis LA, McCourt RM. Green algae and the origin of land plants. Am J Bot. 2004:91:1535-56.

7. Přibyl P, Cepák V, Zachleder V. Production of lipids in 10 strains of Chlorella and Parachlorella, and enhanced lipid productivity in Chlorella vulgaris. Appl Microbiol Biotechnol. 2012;94:549-61.

8. Pribyl P, Cepák V, Zachleder V. Production of lipids and formation and mobilization of lipid bodies in Chlorella vulgaris. J Appl Phycol. 2013;25:545-53.

9. Guccione A, Biondi N, Sampietro G, Rodolfi L, Bassi N, Tredici MR. Chlorella for protein and biofuels: from strain selection to outdoor cultivation in a Green Wall Panel photobioreactor. Biotechnol Biofuels. 2014;7:84.

10. Krienitz L, Hegewald EH, Hepperle D, Huss VAR, Rohr T, Wolf M. Phylogenetic relationship of Chlorella and Parachlorella gen. nov. (Chlorophyta, Trebouxiophyceae). Phycologia. 2004;43:529-42.

11. Guschina IA, Harwood JL. Lipids and lipid metabolism in eukaryotic algae. Prog Lipid Res. 2006:45:160-86.

12. Lang I, Hodac L, Friedl T, Feussner I. Fatty acid profiles and their distribution patterns in microalgae: a comprehensive analysis of more than 2000 strains from the SAG culture collection. BMC Plant Biol. 2011;11:124.

13. Ahmad I, Fatma Z, Yazdani SS, Kumar S. DNA barcode and lipid analysis of new marine algae potential for biofuel. Algal Res. 2013;2:10-5.

14. Bock C, Pažoutová M, Krienitz L. Phylogenetic position of Coronastrum ellipsoideum and description of Parachlorella hussii sp. nov. Biologia. 2011;66:585-94.

15. Mizuno Y, Sato A, Watanabe K, Hirata A, Takeshita T, Ota S, Sato N, Zachleder V, Tsuzuki M, Kawano S. Sequential accumulation of starch and lipid induced by sulfur deficiency in Chlorella and Parachlorella species. Bioresour Technol. 2013;129:150-5.

16. Takeshita T, Ota S, Yamazaki T, Hirata A, Zachleder V, Kawano S. Starch and lipid accumulation in eight strains of six Chlorella species under comparatively high light intensity and aeration culture conditions. Bioresour Technol. 2014;158:127-34.

17. Fernandes B, Teixeira J, Dragone G, Vicente AA, Kawano S, Bišová K, Přibyl P, Zachleder V, Vítová M. Relationship between starch and lipid accumulation induced by nutrient depletion and replenishment in the microalga Parachlorella kessleri. Bioresour Technol. 2013;144:268-74.

18. Li X, Přibyl P, Bišová K, Kawano S, Cepák V, Zachleder V, Čížková M, Brányiková I, Vítová M. The microalga Parachlorella kessleri-a novel highly efficient lipid producer. Biotechnol Bioeng. 2013;110:97-107.

19. Radakovits $R$, Jinkerson RE, Fuerstenberg SI, Tae H, Settlage RE, Boore JL, Posewitz MC. Draft genome sequence and genetic transformation of the oleaginous alga Nannochloropsis gaditana. Nat Commun. 2012;3:686. 
20. Adl SM, Simpson AGB, Lane CE, Lukeš J, Bass D, Bowser SS, Brown MW, Burki F, Dunthorn M, Hampl V, Heiss A, Hoppenrath M, Lara E, le Gall L, Lynn DH, McManus H, Mitchell EAD, Mozley-Stanridge SE, Parfrey LW, Pawlowski J, Rueckert S, Shadwick L, Schoch CL, Smirnov A, Spiegel FW. The revised classification of eukaryotes. J Eukaryot Microbiol. 2012;59:429-514.

21. Kilian O, Benemann CSE, Niyogi KK, Vick B. High-efficiency homologous recombination in the oil-producing alga Nannochloropsis sp. Proc Natl Acad Sci USA. 2011;108:21265-9.

22. Converti A, Casazza AA, Ortiz EY, Perego P, Del Borghi M. Effect of temperature and nitrogen concentration on the growth and lipid content of Nannochloropsis oculata and Chlorella vulgaris for biodiesel production. Chem Eng Process Process Intensif. 2009;48:1146-51.

23. Blanc G, Duncan G, Agarkova I, Borodovsky M, Gurnon J, Kuo A, Lindquist E, Lucas S, Pangilinan J, Polle J, Salamov A, Terry A, Yamada T, Dunigan DD, Grigoriev IV, Claverie J-M, Van Etten JL. The Chlorella variabilis NC64A genome reveals adaptation to photosymbiosis, coevolution with viruses, and cryptic sex. Plant Cell. 2010;22:2943-55.

24. Gao C, Wang Y, Shen Y, Yan D, He X, Dai J, Wu Q. Oil accumulation mechanisms of the oleaginous microalga Chlorella protothecoides revealed through its genome, transcriptomes, and proteomes. BMC Genomics. 2014;15:582.

25. Fan J, Cui Y, Wan M, Wang W, Li Y. Lipid accumulation and biosynthesis genes response of the oleaginous Chlorella pyrenoidosa under three nutrition stressors. Biotechnol Biofuels. 2014;7:17.

26. James GO, Hocart CH, Hillier W, Chen H, Kordbacheh F, Price GD, Djordjevic MA. Fatty acid profiling of Chlamydomonas reinhardtii under nitrogen deprivation. Bioresour Technol. 2011;102:3343-51.

27. James GO, Hocart CH, Hillier W, Price GD, Djordjevic MA. Temperature modulation of fatty acid profiles for biofuel production in nitrogen deprived Chlamydomonas reinhardtii. Bioresour Technol. 2013;127:441-7.

28. Mallick N, Mandal S, Singh AK, Bishai M, Dash A. Green microalga Chlorella vulgaris as a potential feedstock for biodiesel. J Chem Technol Biotechnol. 2012;87:137-45

29. Stephenson AL, Dennis JS, Howe CJ, Scott SA, Smith AG. Influence of nitrogen-limitation regime on the production by Chlorella vulgaris of lipids for biodiesel feedstocks. Biofuels. 2010;1:47-58.

30. Economou CN, Aggelis G, Pavlou S, Vayenas DV. Modeling of single-cell oil production under nitrogen-limited and substrate inhibition conditions. Biotechnol Bioeng. 2011;108:1049-55.

31. Boyle NR, Page MD, Liu B, Blaby IK, Casero D, Kropat J, Cokus SJ, HongHermesdorf A, Shaw J, Karpowicz SJ, Gallaher SD, Johnson S, Benning C, Pellegrini M, Grossman A, Merchant SS. Three acyltransferases and nitrogen-responsive regulator are implicated in nitrogen starvationinduced triacylglycerol accumulation in Chlamydomonas. J Biol Chem. 2012;287:15811-25

32. Fan J, Yan C, Andre C, Shanklin J, Schwender J, Xu C. Oil accumulation is controlled by carbon precursor supply for fatty acid synthesis in Chlamydomonas reinhardtii. Plant Cell Physiol. 2012;53:1380-90.

33. Msanne J, Xu D, Konda AR, Casas-Mollano JA, Awada T, Cahoon EB, Cerutti $\mathrm{H}$. Metabolic and gene expression changes triggered by nitrogen deprivation in the photoautotrophically grown microalgae Chlamydomonas reinhardtii and Coccomyxa sp. C-169. Phytochemistry. 2012;75:50-9

34. Yeh K-L, Chang J-S. Nitrogen starvation strategies and photobioreactor design for enhancing lipid content and lipid production of a newly isolated microalga Chlorella vulgaris ESP-31: implications for biofuels. Biotechnol J. 2011;6:1358-66.

35. Chisti Y. Biodiesel from microalgae. Biotechnol Adv. 2007;25:294-306.

36. Radakovits R, Jinkerson RE, Darzins A, Posewitz MC. Genetic engineering of algae for enhanced biofuel production. Eukaryot Cell. 2010;9:486-501.

37. Ahmad I, Sharma AK, Daniell H, Kumar S. Altered lipid composition and enhanced lipid production in green microalgae by introduction of brassica diacylglycerol acyltransferase 2. Plant Biotechnol J. 2015;13:540-50.

38. Brányiková I, Maršálková B, Doucha J, Brányik T, Bišová K, Zachleder V, Vítová M. Microalgae-novel highly efficient starch producers. Biotechnol Bioeng. 2011;108:766-76.

39. González-Ballester D, Casero D, Cokus S, Pellegrini M, Merchant SS, Grossman AR. RNA-seq analysis of sulfur-deprived Chlamydomonas cells reveals aspects of acclimation critical for cell survival. Plant Cell. 2010;22:2058-84.

40. Zhu Z, Zhang S, Liu H, Shen H, Lin X, Yang F, Zhou YJ, Jin G, Ye M, Zou H, Zou H, Zhao ZK. A multi-omic map of the lipid-producing yeast Rhodosporidium toruloides. Nat Commun. 2012;3:1112.

41. Wayama M, Ota S, Matsuura H, Nango N, Hirata A, Kawano S. Threedimensional ultrastructural study of oil and astaxanthin accumulation during encystment in the green alga Haematococcus pluvialis. PLoS One 2013;8:e53618.

42. Ota S, Yoshihara M, Hirata A, Kawano S. 3D-TEM imaging demonstrating dynamic conversion of starch and oil in a cell of Chlorella sorokiniana. Cytologia. 2014;79:287-8.

43. Nguyen AV, Thomas-Hall SR, Malnoë A, Timmins M, Mussgnug JH, Rupprecht J, Kruse O, Hankamer B, Schenk PM. Transcriptome for photobiological hydrogen production induced by sulfur deprivation in the green alga Chlamydomonas reinhardtii. Eukaryot Cell. 2008;7:1965-79.

44. Lomsadze A, Ter-Hovhannisyan V, Chernoff YO, Borodovsky M. Gene identification in novel eukaryotic genomes by self-training algorithm. Nucleic Acids Res. 2005;33:6494-506.

45. Altschul SF, Madden TL, Schäffer AA, Zhang J, Zhang Z, Miller W, Lipman DJ. Gapped BLAST and PSI-BLAST: a new generation of protein database search programs. Nucleic Acids Res. 1997;25:3389-402.

46. Anders S, Huber W. Differential expression analysis for sequence count data. Genome Biol. 2010;11:R106.

47. Matyash V, Liebisch G, Kurzchalia TV, Shevchenko A, Schwudke D. Lipid extraction by methyl-tert-butyl ether for high-throughput lipidomics. J Lipid Res. 2008;49:1137-46.

48. Takeshita T, Takeda K, Ota S, Yamazaki T, Kawano S. A simple method for measuring the starch and lipid contents in the cell of microalgae. Cytologia. 2015:80:475-81.

49. Spurr AR. A low-viscosity epoxy resin embedding medium for electron microscopy. J Ultrastruct Res. 1969;26:31-43.

50. Reynolds ES. The use of lead citrate at high $\mathrm{pH}$ as an electron-opaque stain in electron microscopy. J Cell Biol. 1963;17:208-12.

\section{Submit your next manuscript to BioMed Central and we will help you at every step:}

- We accept pre-submission inquiries

- Our selector tool helps you to find the most relevant journal

- We provide round the clock customer support

- Convenient online submission

- Thorough peer review

- Inclusion in PubMed and all major indexing services

- Maximum visibility for your research

Submit your manuscript at www.biomedcentral.com/submit

C BioMed Central 